\title{
Pathways to brain resilience
}

\author{
Dena B. Dubal is a physician-scientist and the endowed chair in aging and neurodegenerative disease in the \\ neurology department at the University of California, San Francisco. She has received awards from the National \\ Institutes of Health and American Federation for Aging Research.
}

\section{Dena B. Dubal}

am the daughter of a physician and a scientist - and I unwittingly became a physician-scientist. In middle school, my father taught me the wonders of creating blood smears and analyzing urine in his laboratory. Remarkably, these biological samples often revealed a pivotal diagnosis like pregnancy, infection or anemia. A few years later, my mother invited me into the operating room, where I observed, in awe, the cutting into and fixing of a nose, chest or belly. Each surgery repaired a problem. I oddly delighted in the sterile scent of the lab and hospital and cherished these early times of curiosity and marvel with my parents.

As a teenager, however, I wanted little to do with science or medicine. Instead, Zora Neale Hurston, Amy Tan and Ursula Le Guin captured my imagination through their masterful story-telling and provocative challenges to self identity and societal norms. I learned to introspect and write. Alongside this grew an earnest joy in pottery, painting, graphic design and sculpture. I learned to create. And then, as an undergraduate at the University of California, Berkeley, I became an anthropologist. I learned to query human experience across societies and belief systems. So literature, art and culture became integrated into my DNA - and probably set the stage for my robust scientific inquiry and compassionate clinical care.

My molecular backbone, though, was science and medicine. It turned out that my sculptures depicted DNA, and my anthropologic studies focused on culture and disease. I was fascinated that dementia eroded human experience in a different way in rural India compared to urban America. My passion led to a growing focus on the brain itself - and a strong desire to delve deep into its molecular underpinnings. I really wanted to understand and actually do something about the diseases that people across the world suffer, regardless of their culture.

These events led me directly into medicine. I joined the medical scientist training program at the University of Kentucky, where my deep value of human

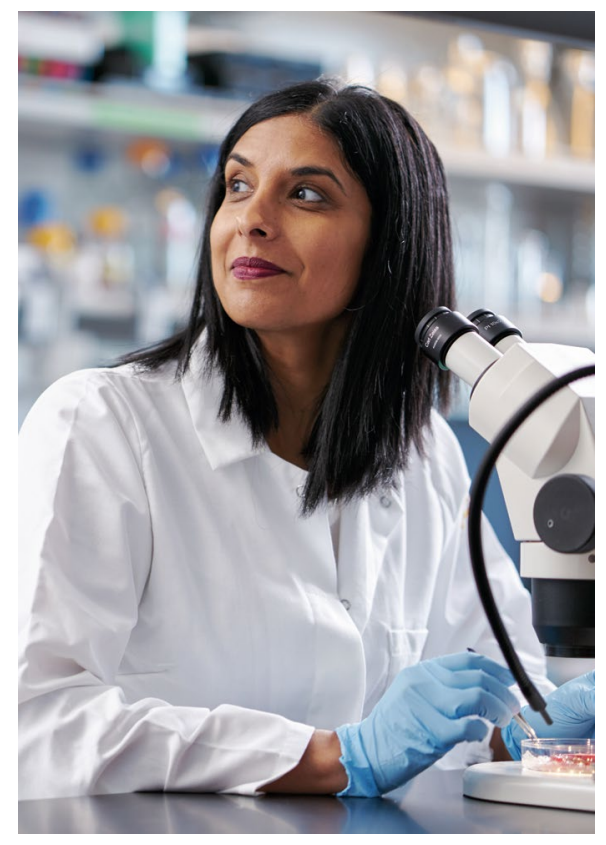

Credit: UCSF

experience integrated with an unrelenting curiosity about neuroscience. I trained with Phyllis Wise, a pioneer and force in neuroendocrinology who ingrained within me a love for scientific discovery and amplified my ambition to move this science toward humans. From there, I matched into a neurology residency at the University of California, San Francisco, with the goal of becoming a masterful clinician. As resident physicians, we cared for several thousand patients with devotion and dedication, leaving no stone unturned. It was immediately striking to me that neurologic illness - Alzheimer's disease, stroke, seizures and multiple sclerosis, to name a few - so often attacked cognition, one's ability to think, communicate and learn. For one of our most valued brain functions, we had no effective medical therapies, and still do not. Inability to treat these deficits was a major shortcoming in our ability to improve life experience. I was determined to try and address this.

"Do things that are big and important not incremental and mediocre. They take the same amount of time." Stephen Hauser (the legendary former chair of neurology at University of California, San Francisco) guided me with words that became my mantra as I built my own laboratory group for scientific discovery. We went for 'big', with a mission to improve brain health in creative ways. Since aging is the biggest risk factor for Alzheimer's, we wondered: could we attack the disease by reversing aging of the brain to promote neural resilience? Since women show cognitive resilience with aging, could we harvest factors from $\mathrm{X}$ chromosomes to make new therapies for men and women?

Resiliency is important in both medicine and cellular biology. Understanding molecular pathways to brain resilience could pave new ways to treat cognitive dysfunction, in a manner that is agnostic to the cause of disease. This may prove useful since many different pathogenic proteins contribute to dementias like Alzheimer's and we will need treatments that counter them simultaneously. For over a decade, serving the underserved at the Zuckerberg San Fransisco General Hospital, I continue to be moved by the ability of many people to endure extremely difficult situations. Their individual resilience inspires me to strive in uncovering molecular pathways that could someday translate, across socioeconomic status and cultural background, into a better quality of life.

\section{Dena B. Dubal ${ }^{1,2}$}

${ }^{1}$ Department of Neurology and Weill Institute of Neurosciences, University of California, San Francisco, San Francisco, CA, USA. ${ }^{2}$ Biomedical and Neurosciences Graduate Programs, University of California, San Francisco, San Francisco, CA, USA. e-mail:dena.dubal@ucsf.edu

Published online: 3 July 2019

https://doi.org/10.1038/s41591-019-0502-7 